\section{CASE OF ABSCESS}

COMMUNICATING WITH

THE CAVITY OF THE CHEST.

By Clemment James Hawkins, Esq.,

Surgeon to the Cheltenham Dispensary.

William Vizard, aged twenty-seven, a stableman, was admitted a dispensary patient July 18,1843 , with symptoms of fever, accompanied with great depression of the vital powers.

In the absence of $\mathrm{Mr}$. Hartley, I visited him, when he gave the following history of himself :-He felt "ill all over" on Sunday, the 16th, but went to work; on the 17th he was much worse, shivered, and felt incapable of the least exertion. Complains of pain in both sides, and in the right axilla, back, and head; has been delirious during the night; countenance pale and anxious; eyes sunk in the orbits, and glistening; great thirst; tongue coated with a thick mucus; pulse 120, and sharp; respiration hurried. The symptoms resembled those I have often witnessed in cases of absorption of animal poison from a punctured wound. I carefully examined his hands, and found two sores on the right thumb, but was assured he had not dressed a diseased horse. He had taken some aperient medicine. I prescribed a solution of tartar emetic with nitrate of potash every third hour.

I was sent for a few hours after my first visit, as he was much worse. The pain in the axilla was intense, and extended to the pectoral muscle; he could only lie on his back, and the exertion of talking caused severe pain in the chest. I found an axillary gland indurated. I was desirous of examining the chest, but found it quite impossible to move him. I added one drachm of Battley's opium to the mixture, and a fomentation and poultice to the axilla.

July 19. Was relieved by the poultice, and has slept at intervals; tongue dry and brown; bowels re. lieved; perspiration intense; pulse 110. Continue the mixture without the opium.

20. Passed a bad night; rery delirious; complains of deep-seated pain in the chest ; integuments covering the great pectoral muscle swollen; severe pain in the forehead. Eight leeches to the head; continue the mixture, with twenty drops of Battley.

23. Inflammation of the skin covering the chest apparent; has cough, and expectoration of thick matter; respiration greatly embarrassed; delirium increased at night; there is a boggy feeling over the right pectoral muscle, and there is a tendency to spreading of the inflammation of the skin; he takes one-sixth of a grain of muriate of morphia, with two of grey mercurial powder, every six hours, and a mixture with carbonate of ammonia in an effervescing state.

27. Has improved in his general health since the last note; his tongue has become moister and cleaner; pulse less rapid; there is an evident sense of deep. seated fluctuation communicated to the fingers. With a view to evacuate matter, I made a deep puncture with a lancet, but was disappointed. I determined to leave the case as it was till the next day, as I had no assistance at hand, in the event of hæmorrhage occurring, and I felt convinced a trifling hæmorrhage would probably produce fatal syncope. To have a pint of porter and a nourishing diet.
28. Says he feels better; I enlarged the puncture, and found the cellular tissue sloughing; a little pus escaped.

29. I made another incision near the nipple, and dissected through the pectoral muscle; immediately about a pint of thick, healthy pus, escaped with some air. During the last four days he has expectorated, or rather gulped up, a large quantity of matter. He takes five grains of sesquicarbonate of ammonia, with half a grain of morphia, every night, with porter, \&c.

It would be tedious and unprofitable to narrate the daily particulars of this case ; it is sufficient to observe that the diffuse cellular inflammation increased, and required extensive incisions to arrest its ravages. Air continued to escape through a fistulous opening till August 29, when the aperture closed; and under the use of quinine, opium, and a generous diet, contrary to my expectations, the poor fellow recovered. The chest was examined by Mr. Hartley and myself on the 30th of August, but we could not detect any respiratory murmur. He was then in a very debilitated state; but as soon as the opening had closed I ordered him to take some iodide of potassium, and to go into the country. He returned much improved, and I discharged him in October quite well, the lung having recovered its functions, excepting over a space of about two inches in circumference. I am perfectly aware that the report of this case is incomplete, inasmuch as the auscultation of the chest was not performed; in fact, it was quite impossible to raise him in bed to get at the back, and the exquisite pain on touching the chest anteriorly prevented our applying the stethoscope.

There is one point in the treatment on which $I$ wish to make a remark. At a remote date, in my anxiety to close the opening in the chest, I suggested the application of a bandage around the chest. This, however, was not put in practice (and I am glad it was not), as Mr. Hartley was averse to it, being of opinion that the functions of the chest would be impeded, and the expansion of the lungs prevented.

\section{INPREQUENCY OF PHTHISIS IN MARSHY DISTRICTS.}

M. Ollivier (d'Angers), at the séance of the Academie de Médicine, Nov. 7, said that M. Nepple's researches in the infrequency of phthisis in marshy districts, were confirmed by what $M$. Brera said some years ago on the rarity of phthisis at Venice, a rarity which he attributed to the emanations of the lagunes. During a sojourn at Venice he endeavoured to verify this fact, and found that in the 1,200 or 1,409 patients admitted into the hospitals of that town in a year, there were only seven or eight cases of phthisis, the rest being for the most part cases of intermittent fever and rheumatism.-Gas. dos Hop., Nov. 9.

A RBMEDY FOR THE VOMITINGS OF BARLY PREGNANT WOMBN. BY M. PITSCHAFT OF BADEN.

M. Pitschaft says he has discovered a remedy for the distressing romitings which generally so much affect women in the early stages of pregnancy. His remedy consists of pills of creosote, henbane powder, and distilled water, one to be taken three times a day.-Gas. des Fop., and Hufeland's Journal. 